\title{
Electrophysical properties of shungites at low temperatures
}

\author{
I. A. Moshnikov, V. V. Kovalevski \\ Institute of Geology, Karelian Research Center, RAS, Petrozavodsk, Russia \\ igorm@krc.karelia.ru, kovalevs@krc.karelia.ru
}

PACS 81.05.U, 91.60.Pn

DOI 10.17586/2220-8054-2016-7-1-214-219

\begin{abstract}
The temperature dependence of electrical conductivity and the shielding effectiveness of shungites have been studied at temperatures ranging from 77 to $300 \mathrm{~K}$. The idiosyncrasies of temperature dependences for measuring electrical parameters were determined. Correlations of the $\pi+\sigma$ plasmon energies with changes in the frequency dependence of shielding effectiveness on temperature were determined.
\end{abstract}

Keywords: shungite rocks, carbon, shielding effectiveness, electrical conductivity.

Received: 20 November 2015

\section{Introduction}

Shungite rocks are ancient carbonaceous volcanic-sedimentary rocks found in Karelia. Shungite rocks consist of a carbon matrix (shungite) with micro- and nano-dispersed mineral components and can be regarded as carbon-mineral composites. On the assumption of the molecular, permolecular and band structure, shungite can be described as a natural nanostructured fullerene-like carbon with some unique physical and chemical properties [1,2].

Electromagnetic properties are of special interest, as they are the most informative and relevant characteristics of carbon materials necessary for modern technologies. These properties of carbonaceous materials are closely related to their band and atomic structures and are directly determined by the degree of graphitization, the size of the crystallites, the length and imperfection of the graphene layers, etc. For a particular carbon-bearing material, some structural characteristics that largely affect its electromagnetic properties can be distinguished. For example, deficiencies and nanotube chirality have a significant influence on their conductivity type [3]. Also, the deficiencies of carbon nanotubes have an influence on their temperature effect for the transition from metallic to a semiconducting type of conductivity [4].

It is also possible to identify some structural characteristics for shungites which have a great effect on their electromagnetic properties. The conductivity type of shungites, for example, can be significantly determined at low currents by the intercalation of elements on the boundaries of graphene layers [5]. Also, the intercalation of carbon materials may have a significant impact on their electromagnetic properties. For example, the intercalation of pyrolytic graphite with copper and oxygen results in a temperature effect for the transition from metallic to a semiconducting conductivity [6].

One of the most sensitive physical properties to band and atomic structure of solids is magnetic susceptibility [7]. The magnetic susceptibility of shungites with different permolecular structures differed significantly at temperatures from 90 to $150 \mathrm{~K}$ [8]. The diamagnetic effect is the greatest for samples with preferable orientation of carbon layers and is not observed for shungites with chaotic layer orientation. 


\section{Experimental}

Shungite rocks with high carbon content (shungites) of different origins from Maksovo, Nigozero, Zazhogino, Chebolaksha and Shunga deposits were selected. The samples were powdered and electrophysical parameters, such as the conductivity and the shielding effectiveness were measured at low and room temperatures. The electrical conductivity $(\sigma)$ was measured in a coaxial line by the E7-8 meter at a frequency of $1 \mathrm{kHz}$. The shielding effectiveness (SE) was determined using a selective microvoltmeter SMV 8.5 and SMV 11 at frequencies ranging from $100 \mathrm{kHz}$ to $1 \mathrm{GHz}$. Cooling of the coaxial line was carried out by liquid nitrogen. For comparison, the same measurements were performed for pyrolytic graphite, pyrobitumen of the "Iron river" deposit and carbonaceous matter of "Erickson gold mine" deposit. The Philips 400 FEG electron microscope with Gatan PEEL spectrometer was used to reveal the $\pi+\sigma$ plasmon energies of various shungites.

The electrical conductivity-temperature dependence of shungite from the Shunga deposit has been defined as a nonmonotonic function. First, the electrical conductivity decreases as temperature increases from 130 to $180 \mathrm{~K}$, which is remarkable for metals, and then it is increases from 220 to $260 \mathrm{~K}$, which is typical for semiconductors (Fig. 1). The same effect was also observed for carbonaceous matter from the Erickson deposit with carbon structure similar to that of shungites.

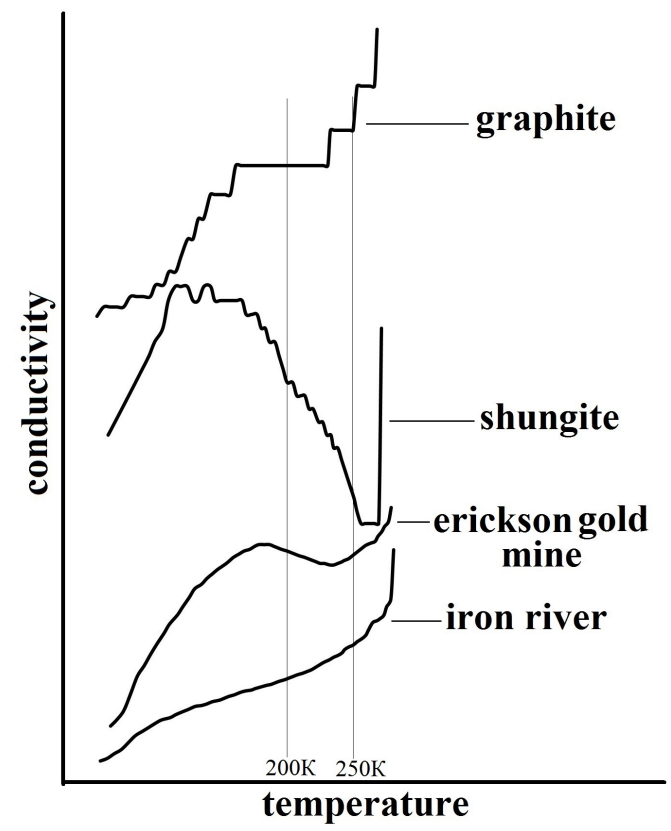

FIG. 1. The electrical conductivity-temperature dependence of pyrolytic graphite, shungite, pyrobitumen of the Iron river deposit and carbonaceous matter of Erickson deposit

Cooling of all samples by liquid nitrogen generally leads to lower values of the shielding effectiveness over the entire measured frequency range. Thus, a slower increase in the shielding effectiveness with electromagnetic field frequency was observed at temperatures less than room temperature. The revealed frequency dependence of shielding effectiveness as function of temperature is different for various shungite rocks as shown in Fig. 2.

To quantify the observed effect, the value $\Delta$ as a rate of change of the shielding effectiveness against frequency has been calculated. Those values as a result of the shielding 


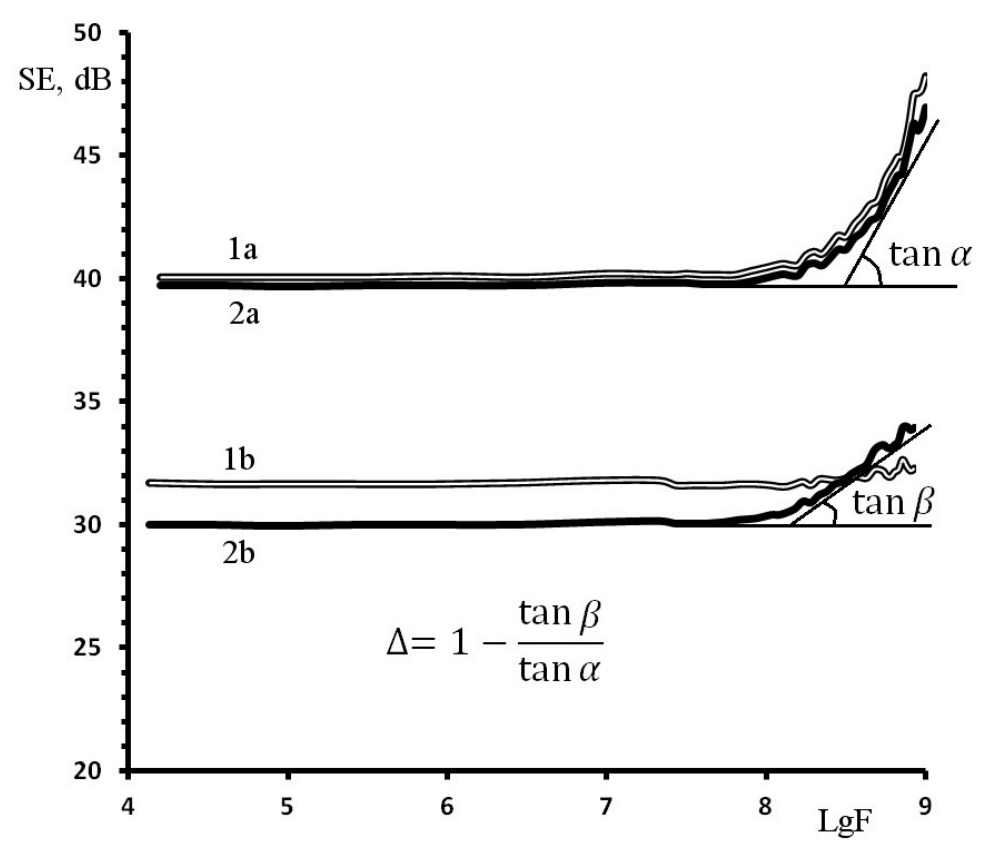

FIG. 2. Dependence of shielding effectiveness of shungite rocks on electromagnetic field frequency. 1a - deposit Maksovo $T \sim 300 \mathrm{~K}, 1 \mathrm{~b}-$ deposit Maksovo $T \sim 77 \mathrm{~K}, 2 \mathrm{a}$ - deposit Shunga $T \sim 300 \mathrm{~K}, 2 \mathrm{~b}-$ deposit Shunga $T \sim 77 \mathrm{~K}$

TABLE 1. The shielding effectiveness (SE), the $\Delta$ values and the $\pi+\sigma$ plasmon energies for various shungites

\begin{tabular}{|c|c|c|c|c|}
\hline Deposit & SE $(750 \mathrm{MHz}, 300 \mathrm{~K}), \mathrm{dB}$ & $\mathrm{SE}(750 \mathrm{MHz}, 77 \mathrm{~K}), \mathrm{dB}$ & $\Delta$ & Energy $\pi+\sigma, \mathrm{V}$ \\
\hline Maksovo & 48 & 32 & 0.55 & 24.9 \\
\hline Nigozero & 46 & 31 & 0.60 & 25.1 \\
\hline Zazhogino & 60 & 42 & 0.70 & 25.3 \\
\hline Chebolaksha & 48 & 33 & 0.90 & 26.3 \\
\hline Shunga & 49 & 32 & 0.95 & 26.0 \\
\hline
\end{tabular}

effectiveness - frequency change at different temperatures ( $\Delta$ in Fig. 2) have a fairly good correlation with the $\pi+\sigma$ plasmon energies of shungites, which are responsible for the bond strengths of valence electrons (Table 1). Namely, as can be deduced from the table, the higher the energy, the slower the shielding effectiveness growth with frequency at lower temperatures.

Studies of the shielding effectiveness at $750 \mathrm{MHz}$ as a function of temperature (ranging from $77 \mathrm{~K}$ to $300 \mathrm{~K}$ ) showed that the change in the shielding effectiveness is not monotonic for the studied shungites. At least three differing regions of the function occur for the shungites and carbonaceous matter from the gold Erickson deposit. An increase in the shielding effectiveness from $77 \mathrm{~K}$ to about $180 \mathrm{~K}$ was observed, then in the $180-200 \mathrm{~K}$ temperature range, it decreases, and at about $230-250 \mathrm{~K}$ temperature it once again increases. Similar variations for the shielding effectiveness versus temperature were not observed for graphite and pyrobitumen from the Iron river deposit, where the shielding efficiency increases for those materials were more monotonous (Fig. 3). 


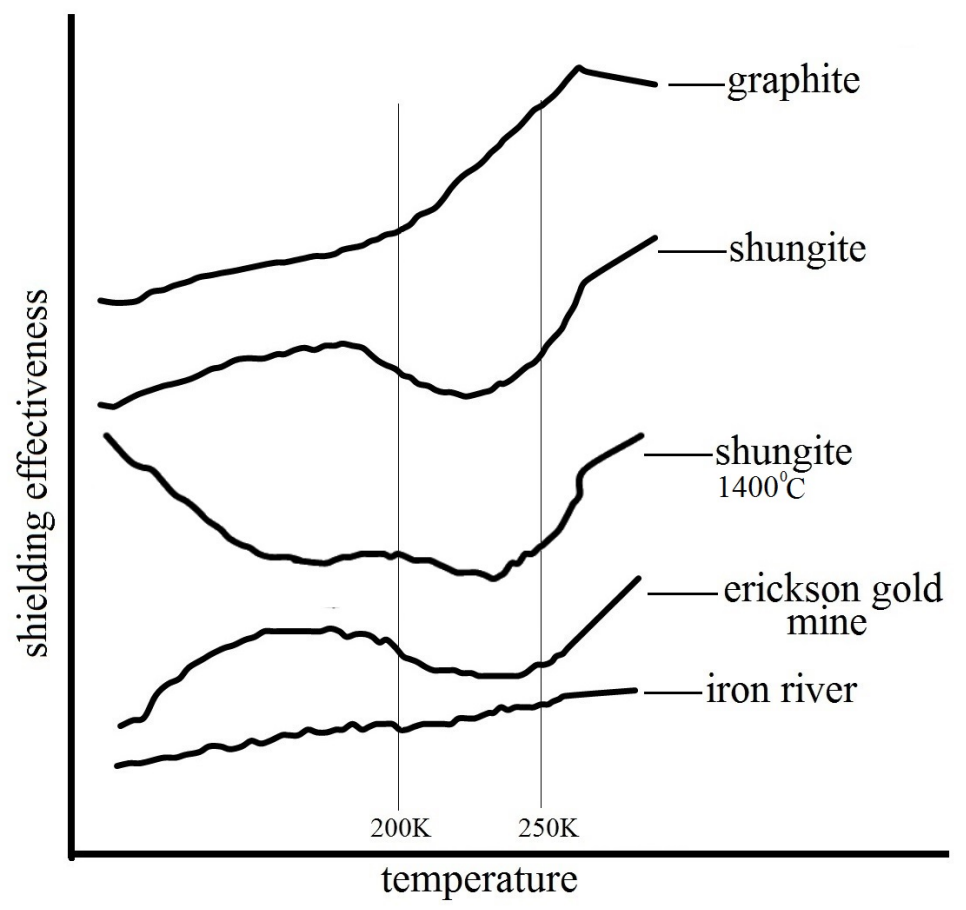

FIG. 3. Temperature dependence of the shielding effectiveness for pyrolytic graphite, shungite, heat-treated $\left(1400^{\circ} \mathrm{C}\right)$ shungite, pyrobitumen of the Iron river deposit and carbonaceous material of the Erickson deposit

To define the possible effect of bound water on the shielding effectiveness growth function, similar studies of the heat-treated shungite $\left(1400{ }^{\circ} \mathrm{C}\right)$ have been performed. The $1400{ }^{\circ} \mathrm{C}$ boundary temperature is one that leads to complete water extraction without causing any structural change to the shungite carbon. The shielding effectiveness at $750 \mathrm{MHz}$ from 77 to $300 \mathrm{~K}$ for the heat-treated shungite has demonstrated a function similar to that of untreated shungite (Fig. 3). This result provides evidence against water having an effect on the shielding effectiveness growth function.

\section{Discussion of the results}

Changes in electrical conductivity as a function of temperature obtained in our experiments is similar to that described in [9] for semiconductors, in which the charge carrier can be small radius polarons (Fig. 4). Based on the presented model, it is shown that at low temperatures electrons are mainly related to the donors (area 1). At temperatures below $1 /{ }_{2} \Theta$ (where $\Theta$ is the Debye temperature) an electron transfers a charge like a heavy particle, being scattered by impurities or lattice vibrations, so the electrical conductivity decreases with increasing temperature (area 2). At temperatures above $1 / 2 \Theta$ the electrical conductivity becomes hopping and increases with temperature increasing (area 3) [9]. Based on values of $\Theta$, determined from the heat capacity curves for Shunga and Maksovo shungites, respectively 420 and $530 \mathrm{~K}$ [10], the temperature ranges over which the effect was observed in our experiment and was described in [9] are similar. An anomaly of the temperature dependence of the electrical conductivity has been reported for shungites as a jump in its growth at about $180 \mathrm{~K}$ [11]. The effect was accounted for by the occurrence of fullerenes in shungites. In our studies, a jump in the growth of the shungites' electrical conductivity was not observed, rather a smooth change occurred over a similar temperature range. 


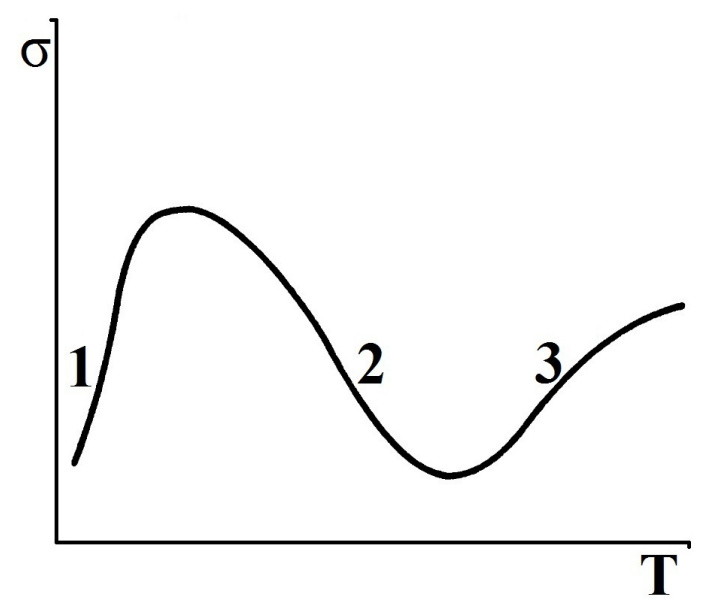

FIG. 4. Typical dependence of electrical conductivity on temperature for a crystalline semiconductor, when the carriers are polarons [9]

We believe that, the behavioral features of the electrical characteristics revealed in our experiments may be a result of many factors. In addition to the influence of structural parameters, such factors as shape and condition of the surface of particles, the presence of moisture and other contact phenomena may also have an effect. The fact that temperature relationships with electrical conductivity and shielding effectiveness were observed only for shungites and Erickson carbonaceous matter (which is similar to shungite in structure) provides evidence for the carbon structure's influence on the effects. The correlation of energy values for the $\pi+\sigma$ plasmon with a change in the frequency dependence of shielding effectiveness on temperature indicates the participation of valence electrons in the shielding mechanism. The similarity of the electrical conductivity dependency on temperature for shungite, and that described for semiconductor materials, in which charge carriers can be polarons, suggests that the detected temperature effect may be associated with the mechanism for the interaction of phonons with charge carriers. Thus, it can be noted that the presence of such temperature effects may be associated with structural features of the shungite carbon, and with the presence of trace elements and non-periodic structural defects in shungite carbon layers, specifically.

\section{Acknowledgements}

This work supported in part by the RFBR grant 13-05-98811.

\section{References}

[1] Kovalevski V.V. Shungite or higher anthraxolite? Zapiski RMO, 2009, 5, P. 97-105.

[2] Augustyniak-Jablokow M.A., Yablokov Y.V., Andrzejewski B., Kempinski W., Los S., Tadyszak K., Yablokov M.Y., Zhikharev V.A. EPR and magnetism of the nanostructured natural carbonaceous material shungite. Phys. Chem. Minerals, 2010, 37(4), P. 237-247.

[3] Bobenko N.G., Egorushkin V.E., Melnikova N.V., Ponomarev A.V. Low-temperature electrical resistivity in carbon nanotubes. Proc. "Carbon: fundamental problems of science, material science, technology". Troitsk, 2012, P. 65-66.

[4] Babaev A.A., Hohlachev P.P., Terukov E.I., Nikoaev Yu.A., Frendin A.B., Filippov R.A., Filippov A.K. The temperature dependence of the resistivity of the composite films on the basis of multi-walled carbon nanotubes. Phys. Solid State, 2015, 55(2), P. 404-407.

[5] Golubev E.A. Electrophysical properties and structural features of shungite. Phys. Solid State, 2013, 55(5), P. 995-1002. 
[6] Masterov M.A., Prichodko A.V., Konkov O.I. The metal-insulator transition and superconductivity in allotropes of carbon intercalated with copper: prediction and experiment. Fuller. Sci. Technol., 1998, 6(3), P. 481-497.

[7] Heremans J., Olk C.H., Morelli D.T. Magnetic susceptibility of carbon structures. Phys. Rev. B, 1994, 49(21), P. 15122-15125.

[8] Kovalevski V.V., Prikhodko A.V., Buseck P.R. Diamagnetism of natural fullerene-like carbon. Carbon, 2005, 43, P. 401-405.

[9] Mott N.F., Davis E.A. Electron processes in non-crystalline materials. Mir, M, 1982, P. 107-108.

[10] Tupolev A. G., Dukiev E. F. Heat capacity. The shungit - a new carbonaceous matter. Petrozavodsk, 1984, P. 84-89.

[11] Parfenjeva L.S., Smirnov I.A., Zaydenberg, A.Z., Rozhkova N.N., Stefanovich, G.B. Electrical conductivity of shungite carbon. Phys. Solid State, 1994, 36(1), P. 234-236. 